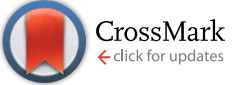

Cite this: RSC Adv., 2017, 7, 7954

Received 15th November 2016 Accepted 17th January 2017

DOI: 10.1039/c6ra26807b

www.rsc.org/advances

\section{Preparation and characterization of electrospun graphene/silk fibroin conductive fibrous scaffolds}

\begin{abstract}
Yi Yang,$\uparrow^{a}$ Xili Ding,$\uparrow^{a}$ Tongqiang Zou, ${ }^{a}$ Ge Peng, ${ }^{a}$ Haifeng Liu ${ }^{\star a}$ and Yubo Fan ${ }^{\star a b}$
Electroactive scaffolds which can carry electrical stimulation to the cells growing on them have attracted more and more attention in recent years. In this study, a conductive fibrous scaffold made of silk fibroin (SF) and graphene was developed using electrospinning techniques. The chemical structural characterization of the obtained scaffolds confirmed the presence of graphene in the fibrous scaffolds. The surface morphologies, mechanical and electrochemical properties and cytocompatibility of the scaffolds were evaluated. The average diameters of the G/SF fibrous scaffolds increased with the addition of graphene until the content of graphene reached $4 \%$. The G/SF scaffolds exhibited improved thermal stability with the addition of graphene, which confirmed that they were more crystalline than pure SF scaffolds. The 3\% G/SF fibrous scaffolds showed improved electroactivity and mechanical properties. In addition, they could support the growth and expansion of rat bone mesenchymal stem cells (rBMSCs) based on cell morphology, viability and proliferation studies in vitro. Thus, all the data in this study suggested that the $3 \% \mathrm{G} / \mathrm{SF}$ scaffolds might represent an adequate substrate to successfully scaffold electroactive tissue during regeneration or engineering.
\end{abstract}

\section{Introduction}

Silk fibroin (SF) has been used as an attractive biomaterial for tissue regeneration, ${ }^{1}$ which features excellent cytocompatibility, adaptable biodegradability, and good oxygen/water vapor permeability. ${ }^{2-4}$ Additionally, it can be processed easily into various structures, such as meshes, membranes, hydrogels, sponges, and fibers. ${ }^{1,4}$ SF fibrous scaffolds created by electrospinning techniques have enormous potential for tissue regeneration since they can mimic the structure and function of native extracellular matrix (ECM). ${ }^{5}$ Moreover, the electrospun fibers have a large surface area to volume ratio, which allows for the direct attachment of ECM ligands, growth factors, and other biomolecules onto the fiber surfaces to locally modulate cell and tissue function and to enhance tissue regeneration. ${ }^{6,7}$ However, the field of potential applications could be tremendously expanded by developing electrospun scaffolds with conductive properties since life involves chemical reactions and electrical currents. ${ }^{\mathbf{8} 9}$ Electrical stimulation of cells and tissues is widely used to enhance their different biological functions.

${ }^{a}$ Key Laboratory for Biomechanics and Mechanobiology of Ministry of Education, International Research Center for Implantable and Interventional Medical Devices, School of Biological Science and Medical Engineering, Beihang University, Xue Yuan Road No. 37, Haidian District, Beijing 100191, People's Republic of China. E-mail: haifengliu@buaa.edu.cn; yubofan@buaa.edu.cn; Fax: +86-10-82338456; +86-1082339428; Tel: +86-10-82338456; +86-10-82339428

${ }^{b}$ National Research Center for Rehabilitation Technical Aids, Beijing 100176, People's Republic of China

$\dagger$ The two authors contributed equally to this work.
Some cells, such as cardiomyocytes, neurons, and skeletal muscle cells, are electroactive and respond directly to electrical impulses. ${ }^{\mathbf{8 1 0}}$ Herein, there is a permanent demand for improved electroactive scaffolds, which can carry electrical stimulation to the cells growing on them. ${ }^{10}$

Graphene, an atomically thick, two dimensional sheet composed of $\mathrm{sp}^{2}$ carbon atoms arranged in a honeycomb crystal lattice, ${ }^{\mathbf{1 1}}$ has attracted a lot attention in recent years due to its outstanding properties such as excellent thermal and electrical conductivity, high Young's modulus, large specific surface area, and biocompatibility. ${ }^{12,13}$ Graphene has been developed for numerous potential applications in biomedicine, such as disease diagnostics, antibacterial and antiviral materials, biosensing, cancer targeting and photothermal therapy, electrical stimulation of cells, drug delivery, and tissue regeneration. ${ }^{\mathbf{1 2 - 1 6}}$ As we all know, the high electrical conductivity of graphene is due to its unique structure and strong $\mathrm{C}=\mathrm{C}$ bonding. Low defect density in the crystal lattice imparts single-layered graphene excellent electrical conductivity. ${ }^{17}$ Moreover, conductive graphene can significantly improve the electrical conductivity of the composites when being used as fillers with insulating polymer matrix. For example, graphene/polyvinylpyrrolidone composites were produced by Wajid et al. using bulk polymerization of $N$-vinylpyrrolidone loaded with dispersed graphene, which resulted in improved electrical properties. ${ }^{18}$ In another study, Khan and colleagues fabricated the electrospun polyacrylonitrile (PAN) and polymethyl methacrylate (PMMA) nanofiber separators embedded with graphene nanoflakes, and the ionic conductivity were increased from $3.31 \times 10^{-4}$ to 5.52 
$\times 10^{-4} \mathrm{~S} \mathrm{~m}^{-1} \cdot{ }^{19}$ Thus, it could be relevant to confer SF fibrous scaffolds electroconductive functionalities with graphene, since SF-conducting fibrous scaffolds with excellent cytocompatibility would carry electrical stimulation to the cells. ${ }^{20}$

In this study, we present the fabrication of a conductive composite fibrous scaffold made of SF and graphene using electrospinning technique, detecting its surface morphologies, physicochemical and electrochemical properties. Rat bone mesenchymal stem cells (rBMSCs) are cultured on the scaffolds to evaluate the possibilities for their use as supporting electroactive materials for tissue engineering.

\section{Experimental details}

\subsection{Materials}

Raw Bombyx mori silk fibers were supplied from Rugao Chunqiu Textile Co. Ltd (Jiangsu, China). 1,1,1,3,3,3-Hexafluoro-2propanol (HFIP) was purchased from Aladdin Industrial Co. Ltd (Shanghai, China). $\mathrm{Na}_{2} \mathrm{CO}_{3}, \mathrm{CaCl}_{2} \cdot 2 \mathrm{H}_{2} \mathrm{O}$ and $\mathrm{CH}_{3} \mathrm{CH}_{2} \mathrm{OH}$ were obtained from Guangfu Chemical Co. Ltd (Tianjin, China). Graphene was purchased from Xianfeng Tech Co. Ltd. (Nanjing, China).

\subsection{Preparation of SF}

Raw Bombyx mori silk fibers were degummed in a boiling $0.02 \mathrm{M}$ $\mathrm{Na}_{2} \mathrm{CO}_{3}$ aqueous solution for $0.5 \mathrm{~h}$ and then rinsed thoroughly with ultrapure water to purge the sericin from the fibers. And after being dried, the degummed SF was dissolved in a ternary solvent system of $\mathrm{CaCl}_{2} \cdot 2 \mathrm{H}_{2} \mathrm{O} / \mathrm{H}_{2} \mathrm{O} / \mathrm{CH}_{3} \mathrm{CH}_{2} \mathrm{OH}$ solution $(1 / 8 / 2$ in mol ratio) which heated to $78{ }^{\circ} \mathrm{C}$, then constant temperature heating and stirring for $2 \mathrm{~h}$. After that, the resulting solution was dialyzed against ultrapure water using a SnakeSkin Pleated Dialysis Tubing (PIERCE, molecular-weight cut-off (MWCO): 3500 ) at indoor temperature for $24 \mathrm{~h}$. Finally, the SF solution was freeze-dried in a vacuum ambiance for $48 \mathrm{~h}$ so as to form SF sponges and was kept drying for future use.

\subsection{Electrospun graphene/silk fibroin (G/SF) fibrous scaffolds}

A battery of G/SF electrospinning solutions with different mixing volume of graphene were prepared by dispersing them in HFIP at a SF concentration of $10 \%(w / v)$. Graphene was added into the prepared SF solution according to a certain proportion after being exposed to ultrasound for $3 \mathrm{~h}$. And then, the mixed solution was subjected to $24 \mathrm{~h}$ continuous stirring for intensive mixing and preventing graphene aggregation. Detailed components of graphene in electrospinning solutions for each group were listed in Table 1 . Electrospinning was performed with a glass syringe that has $20 \mathrm{~mm}$ inside diameter capped with a $0.9 \mathrm{~mm}$ inside diameter blunt needle. And the glass syringe was loaded with varying contents of graphene in the electrospinning solutions and was fitted up in a syringe pump with a rate of $1.0 \mathrm{~mL} \mathrm{~h}^{-1}$. A voltage of $15 \mathrm{kV}$ generated by a high voltage DC power supply was applied to the blunt needle. While electrospinning, the graphene suspensions dispersed in SF
Table 1 Different contents of graphene (G) in the electrospinning solutions

\begin{tabular}{llll}
\hline & \multicolumn{2}{c}{ Mass in 10 mL HFIP } & \\
\cline { 2 - 3 } Group & SF $(\mathrm{g})$ & $\mathrm{G}(\mathrm{mg})$ & $\begin{array}{l}\text { Solute concentration } \\
\text { of SF (w/v) }\end{array}$ \\
\hline $0 \% \mathrm{G} / \mathrm{SF}$ & 1 & 0 & $10 \%$ \\
$1 \% \mathrm{G} / \mathrm{SF}$ & 1 & 10 & $10 \%$ \\
$2 \% \mathrm{G} / \mathrm{SF}$ & 1 & 20 & $10 \%$ \\
$3 \% \mathrm{G} / \mathrm{SF}$ & 1 & 30 & $10 \%$ \\
$4 \% \mathrm{G} / \mathrm{SF}$ & 1 & 40 & $10 \%$
\end{tabular}

solutions were drawn into fibers and were collected on a grounded aluminum foil-covered collection plate kept at a distance of $15 \mathrm{~cm}$ from the needle tip. And then, the electrospun fibrous scaffolds were treated with $100 \%$ ethanol for 30 min to induce a $\beta$-sheet conformational transition, which resulted in insolubility in water. ${ }^{1}$ All the experiments process were performed at room temperature. And then, the electrospun fibrous scaffolds were vacuum-dried at room temperature for $24 \mathrm{~h}$ and then stored in desiccators.

\subsection{Characterization}

\subsubsection{Surface properties}

Morphological observation. A transmission electron microscope (TEM, HT7700, Japan) was used to determine the locations of graphene particles within the nanofibers. The surface morphology of G/SF fibrous scaffolds were observed under a scanning electron microscope (SEM) (JEOL JSM-5600LV, Japan) with an accelerated voltage of $15 \mathrm{kV}$. Before the observation, the surface of the samples were coated with a sputter coater (BAL-TEC Inc., USA) equipped with a gold target to increase electrical conductivity of the samples. The average diameters of the fibers were gained by measuring 100 randomly selected fibers on the electron micrographs using Image $\mathrm{J}$ (National Institutes of Health, USA).

Hydrophilicity test. The static water surface contact angles were measured using a VCA optima surface analysis system (AST Products, Inc., USA) to determine the hydrophilicity of the G/SF electrospun scaffolds. The droplet of $5 \mu \mathrm{L}$ deionized water was deposited on the surface of the scaffolds. Five samples for each group were measured.

\subsubsection{Chemical structural characterization}

ATR-FTIR analysis. The variation in the structure of the G/SF scaffolds were investigated by attenuated total reflectance Fourier transform infrared spectrometry (ATR-FTIR, Nicolet 560, USA). Every spectrum was acquired in transmission mode with a resolution of $4 \mathrm{~cm}^{-1}$ and a spectral range of 500-4000 $\mathrm{cm}^{-1}$. Three samples for each group were measured.

Raman spectroscopy. Raman spectral analysis is mainly used to identify the molecules symmetry vibration of a material that under test. It is an effective method that could discern the structure and properties of graphene according to the molecular structure of the graphene model. Raman (HORIBA, France) test was implemented using a laser wavelength of $532 \mathrm{~nm}$, and 
a spectral range of $800-3200 \mathrm{~cm}^{-1}$. Three samples for each group were measured.

2.4.3 Thermodynamic properties. The thermodynamic properties of these G/SF electrospun scaffolds were determined by differential scanning calorimetry (DSC) using a SHIMADZU DSC-600 thermal analysis apparatus (SHIMADZU, Japan). About $5 \mathrm{mg}$ of the dried sample for each group was weighed into an aluminum oxide crucible, and the profiles were recorded from room temperature to $200{ }^{\circ} \mathrm{C}$ at a heating rate of $10{ }^{\circ} \mathrm{C} \mathrm{min}{ }^{-1}$ under a nitrogen atmosphere. Three samples for each group were measured.

2.4.4 Mechanical properties. The G/SF electrospun fibrous scaffolds were cut into rectangular samples, and their tensile properties were measured using a Universal Testing Machine (Instron4505, USA). Then the length, the width and the thickness of these samples for testing were input into the computer. A $100 \mathrm{~N}$ maximum load cell was used at a speed of $5 \mathrm{~mm} \mathrm{~min} \mathrm{~m}^{-1}$ in room temperature. After the tensile process, stress-strain curves of the scaffold samples were obtained. Five samples in dry state for each group were tested. According to the stressstrain curves of each sample, relevant mechanical parameters were calculated: tensile strength and Young's modulus. Tensile strength is the stress at break. Young's modulus is the ratio of the stress and the strain at elastic deformation phase.

2.4.5 Electrochemical properties. All the electrochemical experiments were performed in a Metrohm one compartment, three-electrode electrochemical cell connected to an Autolab (CHI660D, Shanghai, China) potentiostat-galvanostat controlled by computer through ECHEM software. The electrolyte, $0.1 \mathrm{M} \mathrm{NaCl}$ aqueous solution, provided electrochemical behaviors similar to that of the biological ambient. As counter electrode a steel plate having $4 \mathrm{~cm}^{2}$ of surface area was used, after polished, cleaned for $15 \mathrm{~min}$ in acetone ultrasonic bath and rinsed with Milli-Q water before every characterization. The working electrode is a G/SF fibrous membrane: $15-17 \mathrm{~mm}$ length, 6-8 mm width and 90-100 $\mu \mathrm{m}$ thickness. The mass of the used meshes ranges from 8 to $10 \mathrm{mg}$. The reference electrode was a Pt/saturated calomel electrode. All the experiments were performed at $22^{\circ} \mathrm{C}$ (room temperature). Three samples for each group were measured.

2.4.6 Cytocompatibility. rBMSCs were obtained from the femurs and tibias of 30 day-old male Sprague-Dawley rats (Peking University Laboratory Animal Center, Beijing, China) as previously described. ${ }^{21-23}$ All experiments involving the use of animals were in compliance with Provisions and General Recommendation of Chinese Experimental Animals Administration Legislation and were approved by Beijing Municipal Science \& Technology Commission (Permit number: SCXK (Beijing) 2012-0001). Briefly, density gradient centrifugation was performed using the percoll technique (Pharmacia, Uppsala, Sweden) to isolate cells from bone marrow. Then, the cells were cultured in Dulbecco's Modified Eagle Medium-Low Glucose (DMEM-LG; Gibco, Grand Island, NY) supplemented with $10 \%$ fetal bovine serum (Gibco, Grand Island, NY) in cell incubator at $37^{\circ} \mathrm{C}$ with $5 \% \mathrm{CO}_{2}$. When primary rBMSCs became near confluent, they were detached from the flasks with trypsinEDTA solution (Invitrogen, CA, USA), and expanded at a splitting rate of $1: 3$. The rBMSCs at passages $3-4$ were used in the following experiments.

All electrospun fibrous scaffolds were sterilized by immersing in $75 \%$ ethanol for $2 \mathrm{~h}$, being exposed to the UV radiation. Then they were rinsed three times with phosphate buffered saline (PBS) to remove ethanol. rBMSCs were seeded at a density of $1.0 \times 10^{5}$ cells per scaffolds onto $0 \% \mathrm{G} / \mathrm{SF}$ to $4 \%$ G/SF scaffolds placing in the wells of a non-treated 6-well culture plate. After pipetting $150 \mu \mathrm{L}$ of cell medium suspension onto the scaffolds, the plates were incubated for $2 \mathrm{~h}$ to allow cell attachment. And then, cell-free medium was added to bring the total well volume to $2 \mathrm{~mL}$. The cell-seeded scaffolds were grown in vitro in a $5 \% \mathrm{CO}_{2}$ incubator at $37{ }^{\circ} \mathrm{C}$ with the medium being replaced every 3 days. The viability of the cells growing on the fibrous membranes was checked using CCK-8 test (Cell Counting Kit-8, Beyotime, China). After completion of the incubation period $(1,4$, and 7 days), the medium in the 6-well plates was replaced with fresh medium containing $10 \%$ CCK-8 solution. These plates were incubated for $2 \mathrm{~h}$, and the absorbances of the reaction medium from each well were measured at $450 \mathrm{~nm}$ using a microplate reader (Thermo Scientific, USA). For each experiment, 5 samples were evaluated.

The cell morphology of the rBMSCs on the scaffolds was examined by confocal microscopy on day 7 . Cells were fixed in $4 \%$ paraformaldehyde, permeabilized with $0.1 \%$ Triton $\mathrm{X}-100$ in PBS and then blocked in 1\% bovine serum albumin. After washing with PBS, the cells were incubated in phalloidin (Sigma-Aldrich, USA) for $30 \mathrm{~min}$ to stain all filamentous actin (Factin) filaments and with 4,6-diamino-2-phenyl indole (DAPI) (Sigma-Aldrich, USA) for $5 \mathrm{~min}$ to label the nuclei at room temperature. Subsequently, cells were observed using an inverted fluorescence microscope (Olympus Inc., Japan).

\subsection{Statistical analysis}

All data were expressed as means \pm standard deviation (SD) for repetitive tests. One-way analysis of variance (ANOVA) statistical analysis was carried out to assess the statistical significance of results between groups. The statistical analysis was performed with the software SPSS (version 19.0) at a confidence level of $95 \%$. Differences were considered to be statistically significant for values of $p<0.05$.

\section{Results and discussion}

\subsection{Surface properties}

The morphology of graphene was characterized by TEM. Fig. 1A showed the deposited graphene structure on the TEM grid. Some areas appeared darker, which indicated that several small fragments of graphene were stacked together. Dispersion of graphene in the SF matrix without re-stacking together is the key to determine the composite performance. Therefore, the graphene powders were dispersed in HFIP via sonication, and the electrospinning solution was prepared by adding SF solution. As shown in Fig. 1B, graphene was identifiable in the TEM images of electrospun fibers. 

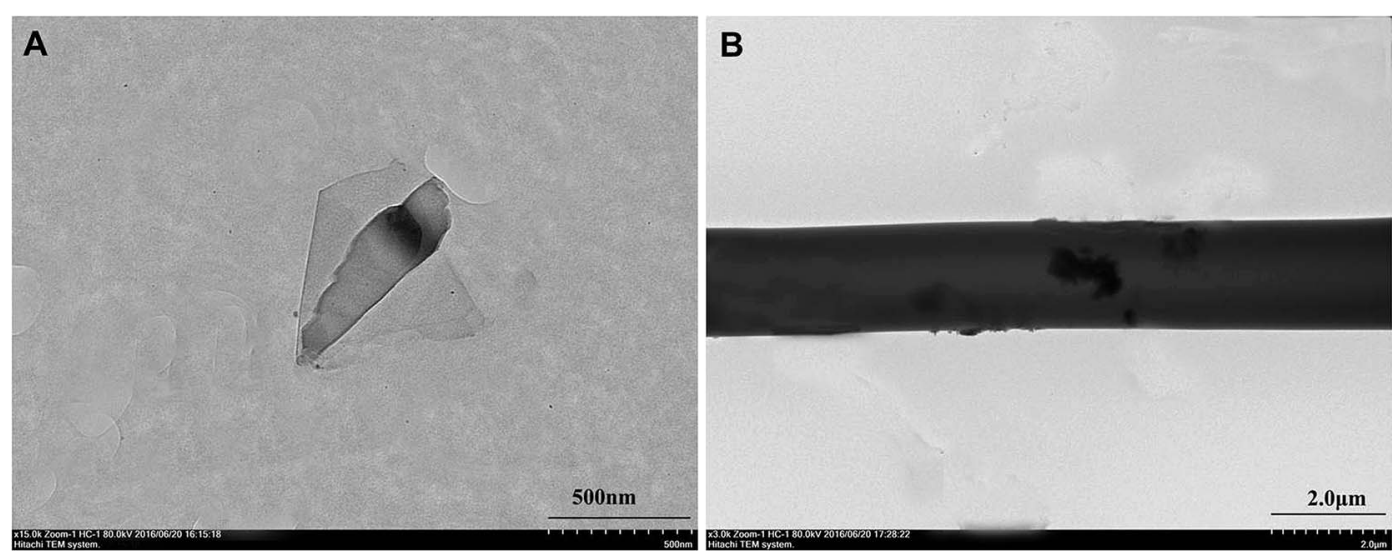

Fig. 1 TEM images of graphene (A) and electrospun G/SF fiber (B).
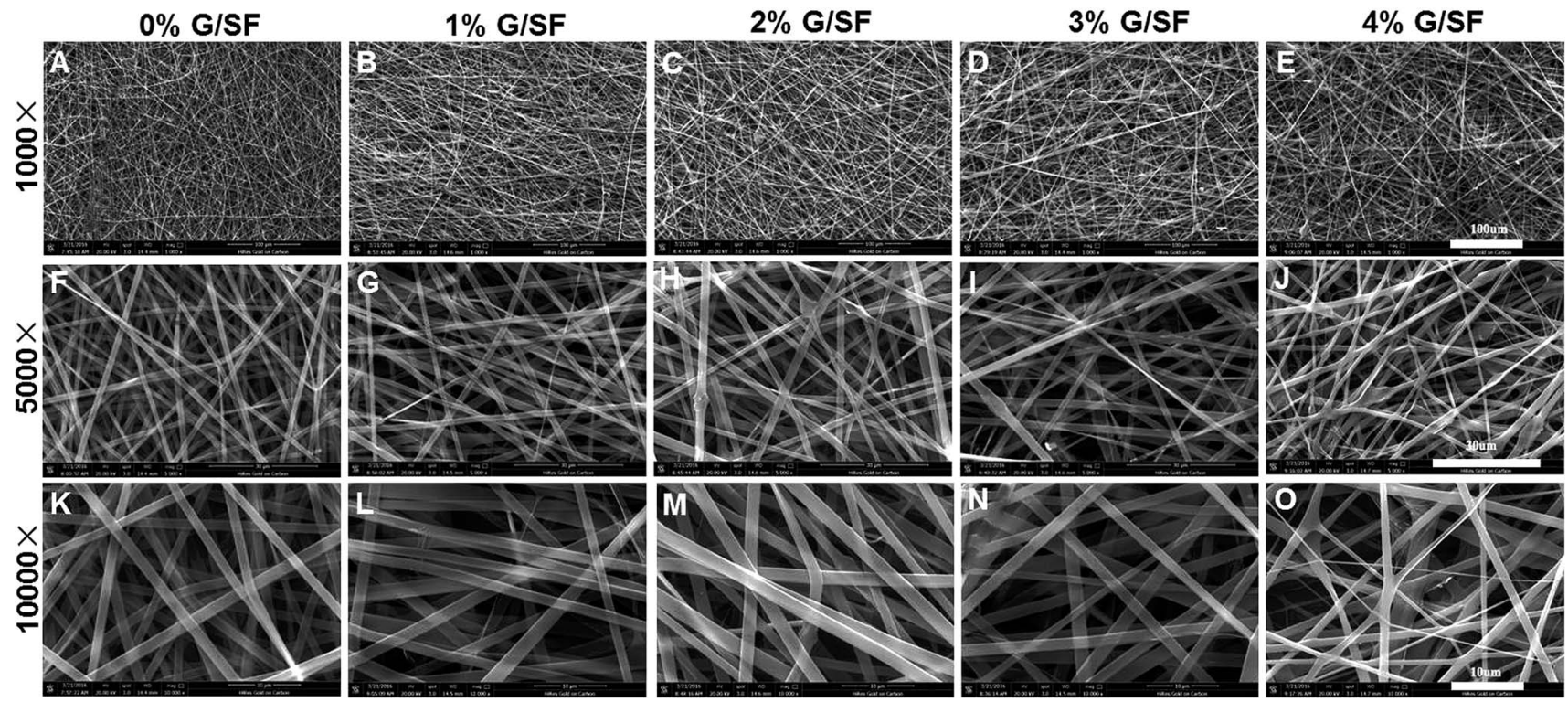

Fig. 2 SEM images of G/SF fibrous scaffolds. 0\% G/SF (A, F and K), 1\% G/SF (B, G and L), 2\% G/SF (C, H and M), 3\% G/SF (D, I and N), 4\% G/SF (E, J and O). (A-E) Scale bars $=100 \mu \mathrm{m}$; $(F-J)$ scale bars $=30 \mu \mathrm{m} ;(K-O)$ scale bars $=10 \mu \mathrm{m}$.

The surface morphologies of G/SF fibrous scaffolds were examined by SEM. As shown in Fig. 2, all of the G/SF fibrous scaffolds exhibited similar surface morphologies. The diameter distributions of the electrospun fibers were shown in Fig. 3. The average fiber diameter of the pure $\mathrm{SF}(0 \% \mathrm{G} / \mathrm{SF})$ scaffolds was smaller than that of all the other groups. Compared with pure SF fibers, the diameter of the fibers showed an increasing trend after adding graphene. The reason could be that the addition of graphene improved the conductivity and the viscosity of the spinning solution, which affected the fiber diameter. In the beginning, the viscosity was considered as the dominant factor of diameter variation. Graphene was easily gathered into flake or graininess in the spinning solution, which led to the enhancement of the viscosity of the spinning system, resulting in the increase of the fiber diameter. However, when the content of graphene reached to $4 \mathrm{mg} \mathrm{mL}^{-1}$, the average diameter of the fibers suddenly decreased. The stretching of the solution mainly owed to the repulsive forces between the charges on the electrospinning jet in the process of electrospinning. ${ }^{24}$ With the increase of the graphene contents, the conductivity became the dominant factor on the variety of fiber diameters. The increased charge density would enhance the elongational forces that exerted on the fiber jet to yield a smaller fiber. ${ }^{25}$ While the graphene contents were too high, graphene flakes could not be fully dispersed in the solution. Moreover, the spinning solution would jam the syringe needle and hinder the jet flow in motion during the electrospinning process, which made the fiber diameter nonuniform (Fig. 2E, $\mathrm{J}$ and $\mathrm{O}$ ).

As shown in Table 2, the contact angles for each group were measured. Besides, while the contact angles were measured, water droplets on the scaffold surfaces were photographed (Fig. 4). The surface of the pure SF scaffolds had a small contact 

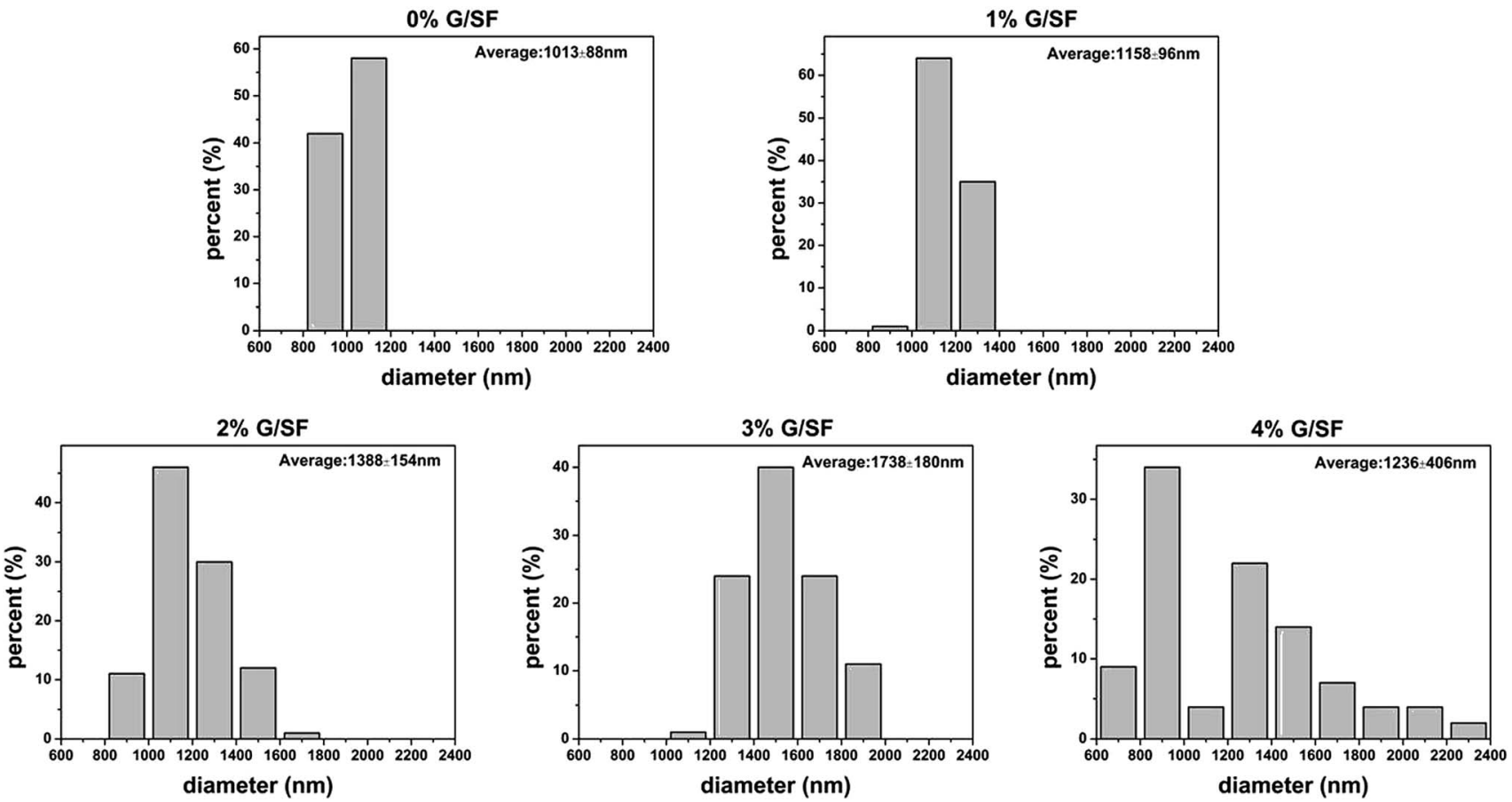

Fig. 3 The average diameters and diameter distributions of G/SF fibrous scaffolds.

Table 2 Static contact angles of G/SF fibrous scaffolds

\begin{tabular}{lll}
\hline Group & $\begin{array}{l}\text { Graphene ratio } \\
\left(\mathrm{mg} \mathrm{mL}^{-1}\right)\end{array}$ & $\begin{array}{l}\text { Static contact } \\
\text { angles }\left(^{\circ}\right)\end{array}$ \\
\hline $0 \% \mathrm{G} / \mathrm{SF}$ & 0 & $21.93 \pm 2.69$ \\
$1 \% \mathrm{G} / \mathrm{SF}$ & 1 & $22.15 \pm 1.16$ \\
$2 \% \mathrm{G} / \mathrm{SF}$ & 2 & $24.01 \pm 2.16$ \\
$3 \% \mathrm{G} / \mathrm{SF}$ & 3 & $28.41 \pm 4.51$ \\
$4 \% \mathrm{G} / \mathrm{SF}$ & 4 & $31.90 \pm 3.78$
\end{tabular}

angle of $21.93^{\circ} \pm 2.69^{\circ}$. For those of $1 \%, 2 \%, 3 \%$, and $4 \%$ G/SF scaffolds, the contact angles increased with the addition of graphene. A smaller contact angle means a more hydrophilic surface of a material. There were a number of hydrophilic groups in SF, such as amine, hydroxyl and carboxyl groups. ${ }^{26}$ However, the graphene layer is hydrophobic. ${ }^{27,28}$ With the addition of graphene in SF, the contact angles showed a gradually increasing trend, i.e., the hydrophobicity of blend fibrous scaffolds increased with the addition of graphene. Many previous studies demonstrated that moderately hydrophilic surfaces $\left(20^{\circ}\right.$ to $55^{\circ}$ water contact angle) benefitted cell attachment and growth on the scaffolds. ${ }^{29-32}$ In this study, the contact angles of all groups were within the suitable range for cell growth.

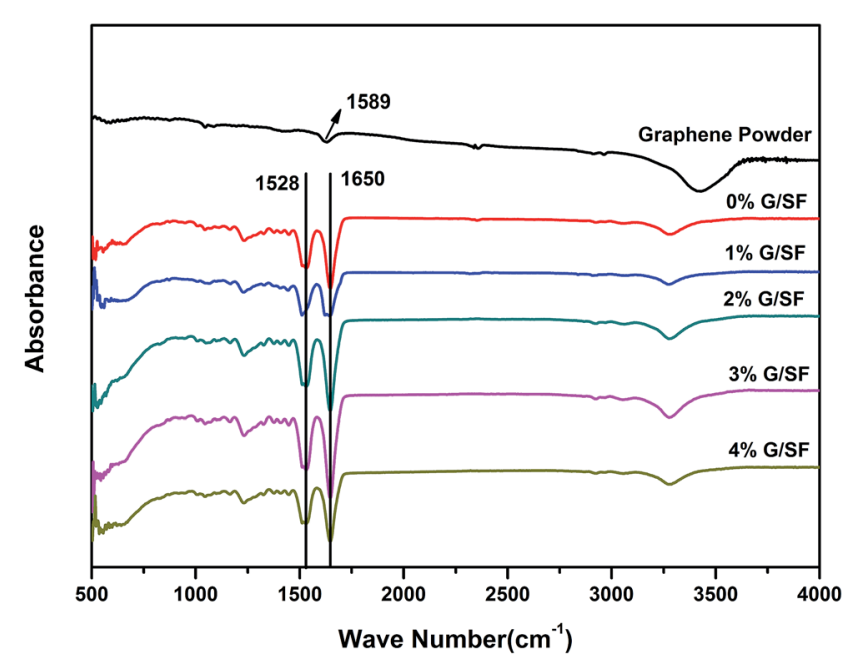

Fig. 5 FTIR spectra of graphene powder and G/SF fibrous scaffolds.

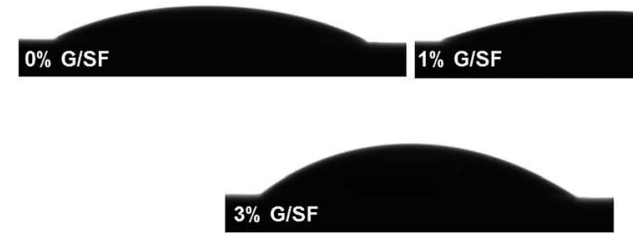

Fig. 4 Photographs of water droplets on G/SF fibrous scaffolds. 


\subsection{Chemical structural characterization}

Fig. 5 showed the ATR-FTIR spectra of G/SF scaffolds prepared with different contents of graphene. The peak at $1589 \mathrm{~cm}^{-1}$ was $\mathrm{C}=\mathrm{C}$ stretching vibration peak for graphene in graphene powder. ${ }^{33}$ Strong absorption bands at $1528 \mathrm{~cm}^{-1}$ and $1650 \mathrm{~cm}^{-1}$ were observed for all G/SF fibrous scaffolds, which could be attributed to the silk-II structure. ${ }^{5,34}$ ATR-FTIR spectra of the $1 \%, 2 \%, 3 \%$, and $4 \% \mathrm{G} / \mathrm{SF}$ fibrous scaffolds did not revealed the band at $1589 \mathrm{~cm}^{-1}$ possibly because the little amount of graphene in SF. The characteristic spectral lines of the composite fibrous scaffolds in $1 \%, 2 \%, 3 \%$, and $4 \% \mathrm{G} / \mathrm{SF}$ had the same absorption peaks as that of pure SF, which showed that the added graphene had no interactions with SF and had no significant effect to the chemical structure of SF. AznarCervantes et al. ${ }^{\mathbf{1 0}}$ obtained similar results for the infrared analysis of SF and graphene composites.

Raman spectroscopy is a powerful nondestructive technique to study carbonaceous materials such as graphene, especially for examining the ordered and disordered crystal structures and also distinguishing the single-, bi-, and multilayer characteristics of graphene and/or graphene oxide layers. ${ }^{35}$ The typical features of carbon materials in Raman spectra are the $\mathrm{G}$ line $\left(\sim 1580 \mathrm{~cm}^{-1}\right)$ and the $\mathrm{D}$ line $\left(\sim 1350 \mathrm{~cm}^{-1}\right)$. In addition, it has been reported that the shape and position of the overtone of $D$ line ( $2 \mathrm{D}$ band at $\sim 2700 \mathrm{~cm}^{-1}$ ) were a notable fingerprint that could be related to the formation and the layer numbers of
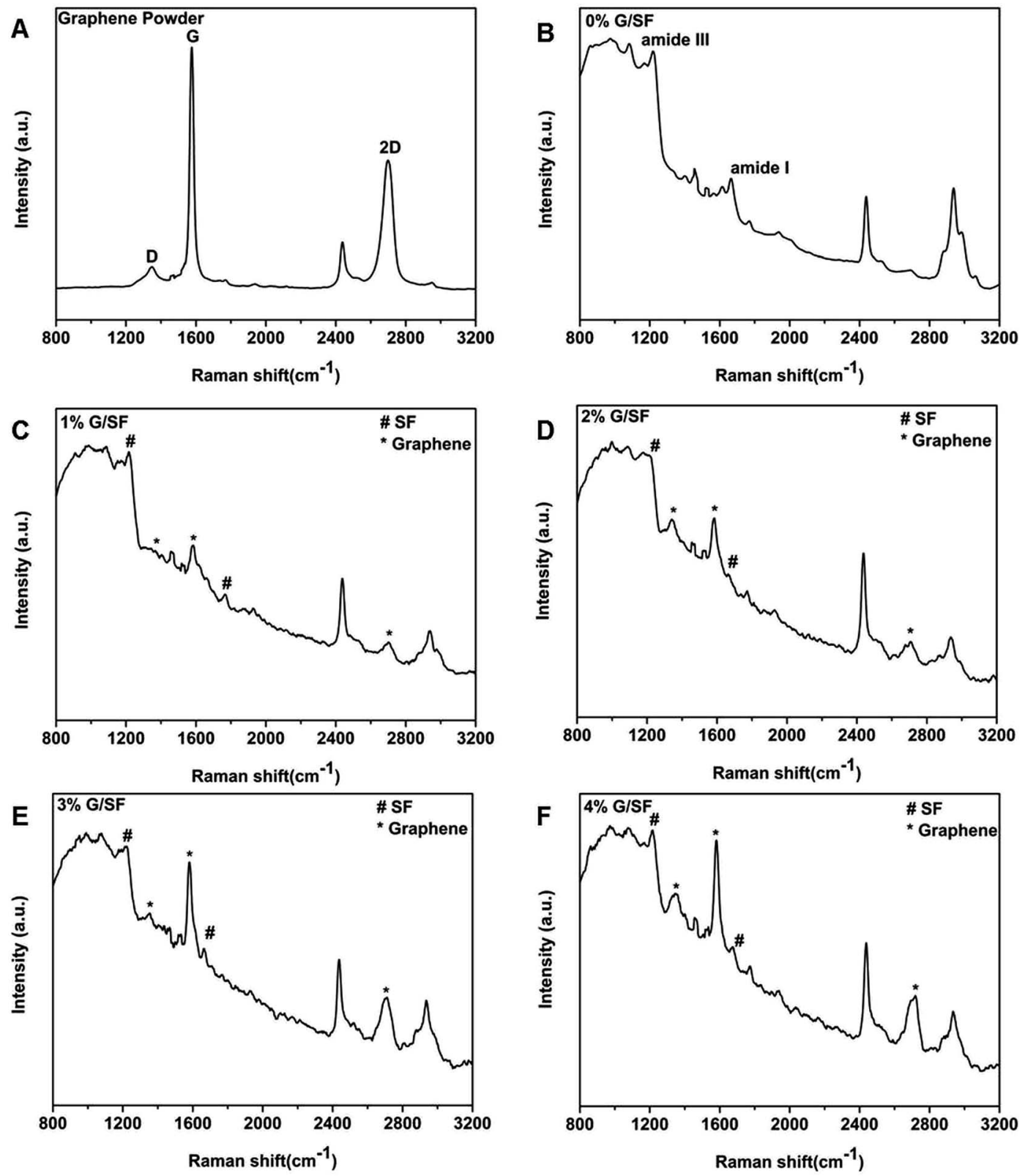

Fig. 6 Raman spectra of graphene powder and G/SF fibrous scaffolds. (A-F) Represents graphene powder, pure SF, 1\% G/SF, 2\% G/SF, 3\% G/SF, and $4 \% \mathrm{G} / \mathrm{SF}$ fibrous scaffolds, respectively. \# Means the characteristic peaks of SF; * means the characteristic peaks of graphene. 
graphene sheets. ${ }^{32}$ In the current study, the value of $1582 \mathrm{~cm}^{-1}$ for the $\mathrm{G}$ band and the peak position of $2 \mathrm{D}$ band located at 2682 $\mathrm{cm}^{-1}$, confirmed the substantial formation of single-layer graphene sheets ${ }^{36-38}$ (Fig. 6A). As shown in Fig. 6B, the strongest bands of $0 \% \mathrm{G} / \mathrm{SF}$ fibrous scaffolds were due to the peptide bonds, including the amide I vibration at $1668 \mathrm{~cm}^{-1}$ (mainly $\mathrm{C}=\mathrm{O}$ stretching vibration), the amide $\mathrm{III}$ vibration at $1230 \mathrm{~cm}^{-1}$ (mainly $\mathrm{N}-\mathrm{H}$ in-plane bending and $\mathrm{C}-\mathrm{N}$ stretching vibrations), ${ }^{39}$ which suggested that the SF scaffolds were predominantly in $\beta$ turn and unordered conformation. ${ }^{40}$ Both the characteristic peaks of graphene and SF were visible from the characteristic spectral line of the fibrous scaffolds (Fig. 6C-F), which demonstrated that graphene was successfully incorporated in the G/SF fibrous scaffolds.

\subsection{Thermodynamic properties}

Thermal behaviors of the G/SF fibrous scaffolds were investigated using DSC in order to understand the influence of graphene on the crystallization of the G/SF fibrous scaffolds. As shown in Fig. 7, the glass transition temperature of the pure SF scaffold was $90{ }^{\circ} \mathrm{C}$. With the addition of graphene, the glass transition temperature of the fibrous scaffolds showed an increasing tendency. Graphene in blended scaffolds might increase the stable reticulation flat structure in the fiber, which

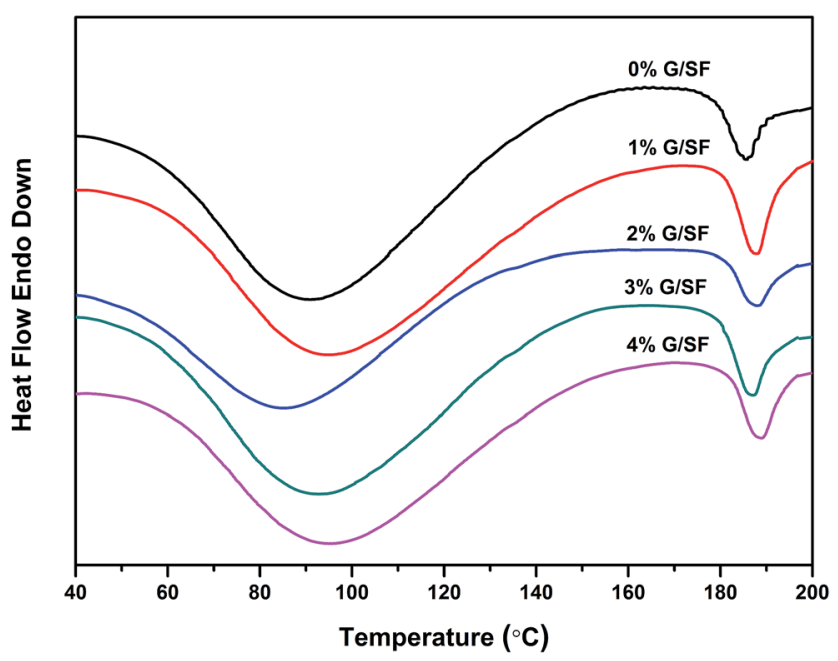

Fig. 7 DSC thermograms of G/SF fibrous scaffolds. made the glass transition temperature of the fibrous scaffolds increased. In addition, the melting temperature of the fibrous scaffolds improved gradually with the addition of graphene. There was an endothermic peak at $183{ }^{\circ} \mathrm{C}$ with the enthalpy of $11.18 \mathrm{~J} \mathrm{~g}^{-1}$ for $0 \% \mathrm{G} / \mathrm{SF}$. For $4 \% \mathrm{G} / \mathrm{SF}$, there was an endothermic peak at $191{ }^{\circ} \mathrm{C}$ with the enthalpy of $59.87 \mathrm{~J} \mathrm{~g}^{-1}$. Larger enthalpy reflected higher degree of crystallization, in other words, G/SF scaffolds were more crystalline than pure SF scaffolds. The polymer molecular chain of the composite fibrous scaffolds was fixed by the hydrogen bond between graphene and SF, which was helpful for crystallizing SF molecules and increasing their melting temperatures.

\subsection{Mechanical properties}

The addition of graphene has been demonstrated to be able to improve the mechanical properties of the composite materials. ${ }^{41,42}$ Thus, we integrated graphene into the SF fibers in the current study to fabricate a G/SF fibrous scaffold with better mechanical properties. The stress-strain curves showed that the mechanical properties of the G/SF electrospun fibrous scaffolds were affected by the contents of graphene (Fig. 8A). The Young's modulus increased from $43.44 \pm 6.81 \mathrm{MPa}$ for $0 \% \mathrm{G} / \mathrm{SF}$ scaffolds to $56.05 \pm 3.42 \mathrm{MPa}$ for $3 \% \mathrm{G} / \mathrm{SF}$ scaffolds (Fig. 8C). Moreover, it was shown in Fig. 8B that the tensile strength gradually improved from $2.01 \pm 0.67 \mathrm{MPa}$ for $0 \% \mathrm{G} / \mathrm{SF}$ scaffolds to $4.28 \pm 1.36 \mathrm{MPa}$ for $3 \% \mathrm{G} / \mathrm{SF}$ scaffolds; i.e., the tensile strength increased by $113 \%$. This might be attributed to the intermolecular forces formed between the SF and graphene, which restricted the movement of the polymer chains. ${ }^{38}$ In addition, as demonstrated by the DSC results, the crystallinity of $\mathrm{G} / \mathrm{SF}$ scaffolds increased with the increase of graphene contents. The crystalline regions might be regarded as crosslinking points of amorphous molecules. The increase of the crystallinity densified the crosslinking points, which could restrain the amorphous molecules from slipping off during the mechanical test. ${ }^{19}$ However, when the graphene content reached $4 \%$, the tensile strength and Young's modulus of the G/ SF scaffolds were lower than those of the pure SF scaffolds (Fig. 8B and C). The reason might be that when further adding graphene to SF solution, graphene would restack together due to van der Waals force of the nanosheets. As mentioned by Zhao et al., there exists a critical point of the mechanical properties upon the graphene nanosheet contents. ${ }^{42}$ Lower than this
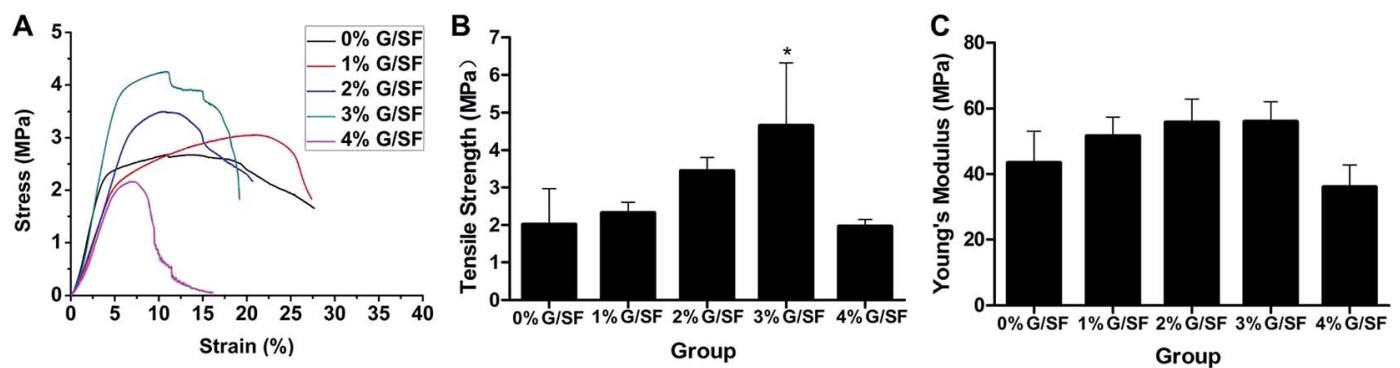

Fig. 8 Mechanical properties of G/SF fibrous scaffolds: (A) stress-strain curves, (B) tensile strength, and (C) Young's modulus. *Indicates significant difference of $p<0.05$. 
content, the exfoliated graphene nanosheets can be welldispersed in the SF, and the increase of graphene content can improve the mechanical properties. However, further increasing of graphene content, higher than this content, may cause the nanosheets stacking together, weakening the mechanical properties of G/SF scaffolds.

\subsection{Electrochemical properties}

The electroactivity of the electrospun G/SF fibrous scaffolds were studied by submitting the scaffolds to voltammetric cycles (50 cycles) in aqueous $0.1 \mathrm{M} \mathrm{NaCl}$. After several consecutive potential cycles (voltammograms between $-0.5 \mathrm{~V}$ and $0.5 \mathrm{~V}$ ), stationary voltammetric responses from the scaffolds with different contents of graphene were shown in Fig. 9. The results

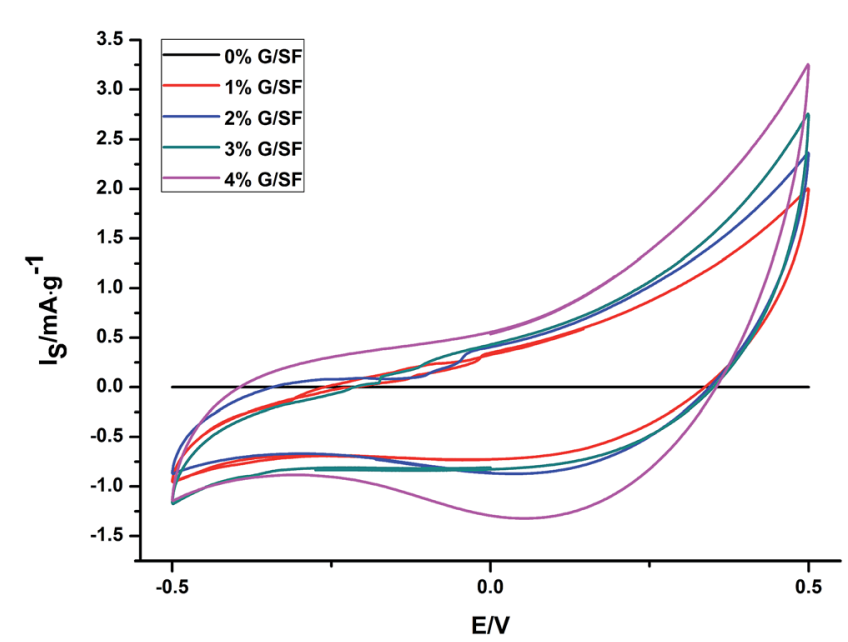

Fig. 9 Stationary voltammetric results of G/SF fibrous scaffolds. showed that the G/SF scaffolds with higher graphene contents exhibited higher currents and thus higher conductivity, which indicated that the G/SF scaffolds could support the flow of a range of anodic and cathodic currents $(< \pm 1.5 \mathrm{~mA})$ in the culture medium and carry electrical stimulation to the cells.

\subsection{Cytocompatibility}

Confocal microscopy analysis of rBMSCs cultured on the G/SF scaffolds revealed their morphological features after culturing for 7 days (Fig. 10). The cells were stained with phalloidin specific for F-actin filaments, and DAPI to visualize the nuclei. The confocal micrographs showed that rBMSCs exhibited spindle shape on all the scaffolds, which meant that the cells spreaded widely on the scaffolds with distinct spread actin filaments. In addition, the viability and proliferation of rBMSCs cultured on the scaffolds were determined by CCK-8 assay. In CCK-8 tests, metabolically active cells react with a tetrazolium salt in the reagent to produce a soluble formazan dye that gives an absorbance at a wavelength of $450 \mathrm{~nm}$. A higher optical density (OD) value signifies better cell viabilities and more live cells. As shown in Fig. 11, an obvious increase of cell proliferation was observed during the 7 day culture in standard conditions, which demonstrated that all samples were nontoxic and rBMSCs were able to adhere and proliferate normally. However, the cell proliferation trend was distinct for different culture times and different scaffolds. The cell viabilities on all the scaffolds increased relatively slowly on day 1 and day 4 , while by day 7 the cell numbers increased sharply. Moreover, there were no significant differences between the viabilities of cells cultured on all the scaffolds on day 1 and day $4(p>0.05)$. However, after 7 days of culturing, the cells on $4 \%$ G/SF scaffolds showed a significant lower proliferation rate than those on the other scaffolds $(p<0.05)$, while no significant differences
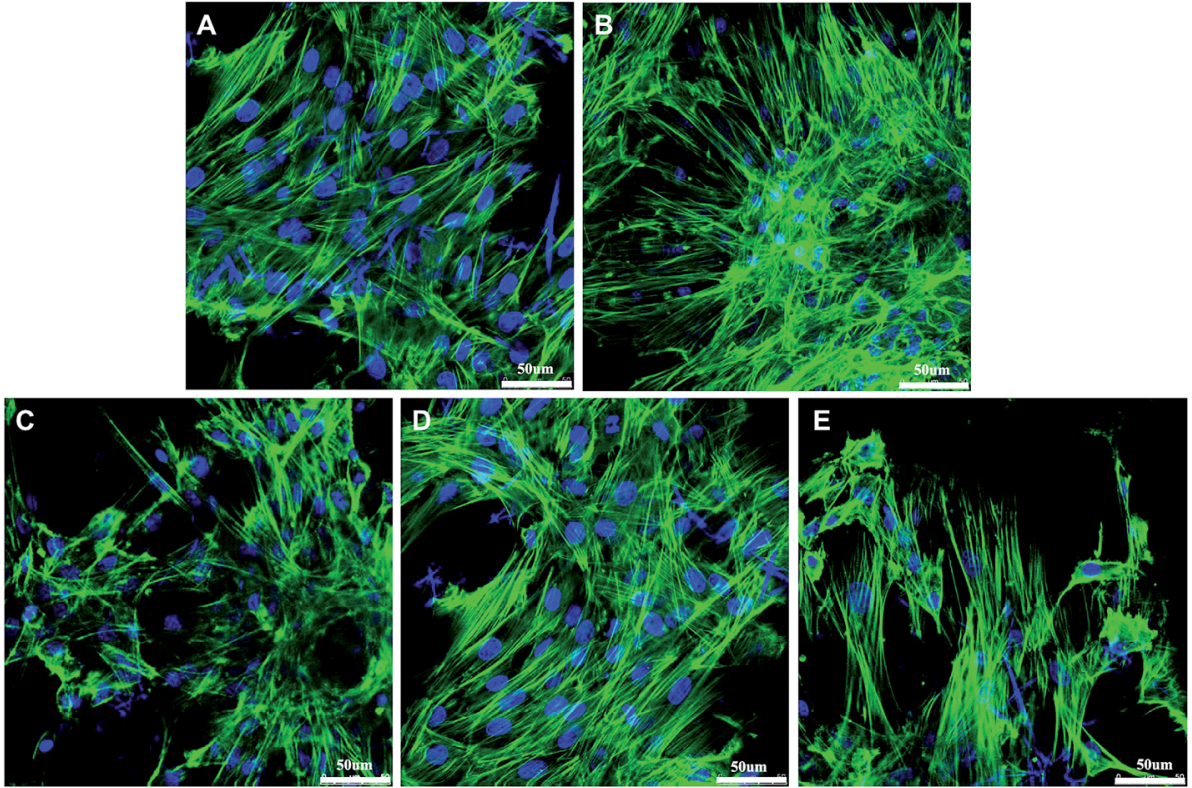

Fig. 10 Immunocytochemistry staining of rBMSCs cultured on G/SF fibrous scaffolds for 7 days. (A-E) Represents $0 \% \mathrm{G} / \mathrm{SF}, 1 \% \mathrm{G} / \mathrm{SF}, 2 \% \mathrm{G} / \mathrm{SF}, 3 \%$ $\mathrm{G} / \mathrm{SF}$, and $4 \% \mathrm{G} / \mathrm{SF}$ fibrous scaffolds, respectively. (A-E) Scale bars $=50 \mu \mathrm{m}$. 


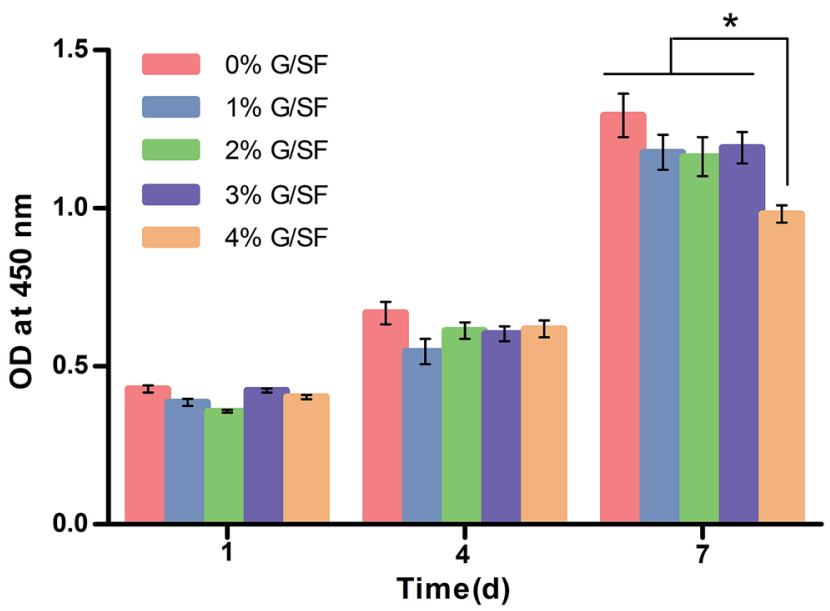

Fig. 11 CCK- 8 assays of rBMSCs cultured on G/SF fibrous scaffolds. "*" Indicates significant difference of $p<0.05$.

were detected between $1 \%, 2 \%, 3 \% \mathrm{G} / \mathrm{SF}$ scaffolds and the pure SF scaffolds $(p>0.05)$. The results indicated that the viabilities and proliferation of rBMSCs on G/SF scaffolds were affected by the contents of graphene.

The results of the in vitro cytocompatibility evaluation demonstrated that the incorporation of graphene did not affect the excellent cytocompatibility of the SF background, but made SF scaffolds become electronic conductors and active. Previous study by Wang et al. indicated that fluorinated graphene (FG) sheets could enhance the neural differentiation of human bone marrow derived mesenchymal stem cell (hBMSC) and the effect could be further enhanced with neuron inducer. ${ }^{43}$ Thus, such properties make the fibrous scaffolds developed in this study (especially the $3 \% \mathrm{G} / \mathrm{SF}$ scaffolds) useful for the application of local electric fields and/or ionic currents to cell culture and differentiation in our future work.

\section{Conclusions}

In this study, a conductive fibrous scaffold made of SF and graphene was developed using electrospinning technique. An increase in the average diameter of the G/SF fibrous scaffolds was observed as the graphene contents in the composite rose till $4 \%$. The $3 \% \mathrm{G} / \mathrm{SF}$ fibrous scaffolds with improved electroactivity and mechanical properties supported the growth and expansion of rBMSCs based on cell morphology, viability and proliferation studies in vitro. Therefore, this could be promising and the $3 \% \mathrm{G} / \mathrm{SF}$ scaffold may allow the potential application of local electric fields or local ionic currents to cell cultures, biological interfaces or animal studies.

\section{Acknowledgements}

This work was supported by the National Natural Science Foundation of China (31470938, 11421202, 61227902, and 11120101001), National Key Technology R\&D Program (2014BAI11B02, 2014BAI11B03， 2016YFC1100704), International Joint Research Center of Aerospace Biotechnology and
Medical Engineering from Ministry of Science and Technology of China, 111 Project (B13003), Research Fund for the Doctoral Program of Higher Education of China (20131102130004), The transformation project for major achievements of Central Universities in Beijing (ZDZH20141000601), Beijing Municipal Science and Technology Project (Z151100003715006), Fundamental Research Funds for the Central Universities.

\section{References}

1 G. H. Altman, F. Diaz, C. Jakuba, T. Calabro, R. L. Horan, J. S. Chen, H. Lu, J. Richmond and D. L. Kaplan, Biomaterials, 2003, 24, 401-416.

2 H. F. Liu, H. B. Fan, Y. Wang, S. L. Toh and J. C. H. Goh, Biomaterials, 2008, 29, 662-674.

3 H. F. Liu, H. B. Fan, S. L. Toh and J. C. H. Goh, Biomaterials, 2008, 29, 1443-1453.

4 H. F. Liu, X. M. Li, X. F. Niu, G. Zhou, P. Li and Y. B. Fan, Biomacromolecules, 2011, 12, 2914-2924.

5 H. F. Liu, X. M. Li, G. Zhou, H. B. Fan and Y. B. Fan, Biomaterials, 2011, 32, 3784-3793.

6 S. Sahoo, L. T. Ang, J. C. H. Goh and S. L. Toh, J. Biomed. Mater. Res., Part A, 2010, 93, 1539-1550.

7 Y. Z. Zhang, J. R. Venugopal, A. El-Turki, S. Ramakrishna, B. Su and C. T. Limet, Biomaterials, 2008, 29, 4314-4322.

8 N. K. Guimard, N. Gomez and C. E. Schmidt, Prog. Polym. Sci., 2007, 32, 876-921.

9 R. Ravichandran, S. Sundarrajan, J. R. Venugopal, S. Mukherjee and S. Ramakrishna, J. R. Soc., Interface, 2010, 7, S559-S579.

10 S. Aznar-Cervantes, J. G. Martinez, A. Bernabeu-Esclapez, A. A. Lozano-Pérez, L. Meseguer-Olmo, T. F. Otero and J. L. Cenis, Bioelectrochemistry, 2016, 108, 36-45.

11 D. Li and R. B. Kaner, Science, 2008, 320, 1170-1171.

12 A. K. Geim, Science, 2009, 324, 1530-1534.

13 M. Kakran and L. Li, Key Eng. Mater., 2012, 508, 76-80.

14 A. K. Geim and K. S. Novoselov, Nat. Mater., 2007, 6, 183-191.

15 K. S. Novoselov, A. K. Geim, S. V. Morozov, D. Jiang, Y. Zhang, S. V. Dubonos, I. V. Grigorieva and A. A. Firsov, Science, 2004, 306, 666-669.

16 H. Y. Mao, S. Laurent, W. Chen, O. Akhavan, M. Imani, A. A. Ashkarran and M. Mahmoudi, Chem. Rev., 2013, 113, 3407-3424.

17 S. Goenka, V. Sant and S. Sant, J. Controlled Release, 2014, 173, 75-88.

18 A. S. Wajid, S. Das, F. Irin, H. S. T. Ahmed, J. L. Shelburne, D. Parviz, R. J. Fullerton, A. F. Jankowski, R. C. Hedden and M. J. Green, Carbon, 2012, 50, 526-534.

19 W. S. Khan, R. Asmatulu, V. Rodriguez and M. Ceylan, Int. J. Energy Res., 2014, 38, 2044-2051.

20 S. Aznar-Cervantes, M. I. Roca, J. G. Martinez, L. MeseguerOlmo, J. L. Cenis, J. M. Moraleda and T. F. Otero, Bioelectrochemistry, 2012, 85, 36-43.

21 X. L. Ding, C. Q. Wu, T. Ha, L. Z. Wang, Y. Huang, H. Y. Kang, Y. Y. Zhang, H. F. Liu and Y. B. Fan, RSC Adv., 2016, 6, 1946319474. 
22 Y. Huang, X. L. Jia, K. Bai, X. H. Gong and Y. B. Fan, Arch. Med. Res., 2010, 41, 497-505.

23 Y. Huang, G. Zhou, L. S. Zheng, H. F. Liu, X. F. Niu and Y. B. Fan, Nanoscale, 2012, 4, 2484-2490.

24 R. Chen, L. J. Qiu, Q. F. Ke, C. L. He and X. M. Mo, J. Biomater. Sci., Polym. Ed., 2009, 20, 1513-1536.

25 X. H. Zong, K. Kim, D. F. Fang, S. F. Ran, B. S. Hsiao and B. Chu, Polymer, 2002, 43, 4403-4412.

26 P. Amornsudthiwat, R. Mongkolnavin, S. Kanokpanont, J. Panpranot, C. S. Wong and S. Damrongsakkul, Colloids Surf., B, 2013, 111, 579-586.

27 S. R. Wang, Y. Zhang, N. Abidi and L. Cabrales, Langmuir, 2009, 25, 11078-11081.

28 H. Li and X. C. Zeng, ACS Nano, 2012, 6, 2401-2409.

29 Y. Yao, H. F. Liu, X. L. Ding, X. J. Jing, X. H. Gong, G. Zhou and Y. B. Fan, J. Bioact. Compat. Polym., 2015, 30, 633-648.

30 K. Webb, V. Hlady and P. A. Tresco, J. Biomed. Mater. Res., 1998, 41, 422-430.

31 J. H. Lee, S. J. Lee, G. Khang and H. B. Lee, J. Colloid Interface Sci., 2000, 230, 84-90.

32 M. Wang, Y. Wang, Y. Chen and H. C. Gu, Appl. Surf. Sci., 2013, 268, 73-78.
33 S. Park, J. An, R. D. Piner, I. Jung, D. X. Yang, A. Velamakanni, S. T. Nguyen and R. S. Ruoff, Chem. Mater., 2008, 20, 6592-6594.

34 C. M. Li, C. Vepari, H. J. Jin, H. J. Kim and D. L. Kaplan, Biomaterials, 2006, 27, 3115-3124.

35 O. Akhavan, Carbon, 2010, 48, 509-519.

36 D. Graf, F. Molitor, K. Ensslin, C. Stampfer, A. Jungen, C. Hierold and L. Wirtz, Nano Lett., 2007, 7, 238-242.

37 A. Dato, V. Radmilovic, Z. Lee, J. Phillips and M. Frenklach, Nano Lett., 2008, 8, 2012-2016.

38 A. C. Ferrari, J. C. Meyer, V. Scardaci, C. Casiraghi, M. Lazzeri, F. Mauri, S. Piscanec, D. Jiang, K. S. Novoselov, S. Roth and A. K. Geim, Phys. Rev. Lett., 2006, 97, 187401.

39 P. Zhou, X. Xie, D. P. Knight, X. H. Zong, F. Deng and W. H. Yao, Biochemistry, 2004, 43, 11302-11311.

40 S. Fan, Y. P. Zhang, H. L. Shao and X. C. Hu, Int. J. Biol. Macromol., 2013, 56, 83-88.

41 M. A. Rafiee, J. Rafiee, Z. Wang, H. H. Song, Z. Z. Yu and N. Koratkar, ACS Nano, 2009, 3, 3884-3890.

42 X. Zhao, Q. H. Zhang and D. J. Chen, Macromolecules, 2010, 43, 2357-2363.

43 Y. Wang, W. C. Lee, K. K. Manga, P. K. Ang, J. Lu, Y. P. Liu, C. T. Lim and K. P. Loh, Adv. Mater., 2012, 24, 4285-4290. 